\title{
Modelling Early-type Stars in Eclipsing Binaries of Open Clusters: A New Method for Age Determination from the Ratio of Radii
}

\author{
M. Yıldız
}

Ege University, Department of Astronomy and Space Sciences, Bornova, 35100 İzmir, Turkey.

Email: mutlu.yildiz@ege.edu.tr

Received 2010 April 2, accepted 2010 October 19

\begin{abstract}
Binary systems, in particular eclipsing binaries, are essential sources of knowledge of the fundamental properties of stars. The ages of binaries, members of open clusters, are constrained by their own fundamental properties and by those of the hosting cluster. The ages of eleven open clusters are here found by constructing models for the components of twelve eclipsing binaries. The difference between the ages we find and the ages of the clusters derived from isochrone fitting is up to $40 \%$. For the binary system V497 Cep in NGC 7160, the difference is about $100 \%$. Binary systems whose primary component is about to complete its main-sequence lifetime, such as V453 Cyg and V906 Sco, are the most suitable systems for age determination. Using model results for these stars, we derive an expression for sensitive and uncomplicated relative age determination of binary systems (age divided by the main-sequence lifetime of the primary star). The expression is given as a logarithm of radii ratio divided by a logarithm of mass ratio. Two advantages of this expression are that: (i) it is nearly independent of the assumed chemical composition of the models because of the appearance of the ratio of radii; and (ii) the ratios of radii and masses are observationally much more precise than their absolute values. We also derive another expression using luminosities rather than radii and compare results.
\end{abstract}

Keywords: binaries: eclipsing — open clusters — stellar: interior — stellar: evolution

\section{Introduction}

Nature is full of signals displaying its past and present structure. Astrophysics is extremely specialized in detecting and extracting such signals from very noisy backgrounds. In this context, research on deep regions of stars is a real adventure. Our knowledge of the internal structure and evolution of stars is mostly based on model computations, which take into account and combine outcomes of almost all branches of modern physics. To test how well our stellar models represent real stars, very accurate observational constraints, such as mass, luminosity, radius, chemical composition, and rotational properties, are required. These constraints are obtained from observation of the outer regions of stars, which depend on the physical conditions in the deep interior.

Time is one of the essential components of our universe. Understanding the evolution of the far and near Universe depends on how successful we are in determination of the ages of astrophysical objects. In this respect, every type of age determination is crucial for completing the picture. In this context, we consider the observed detached eclipsing binaries (DEBs) that are members of open clusters, to obtain some basic information pertaining to stars.

In the literature, thirteen well-observed DEBs in twelve open clusters are found. Among these systems, V818 Tau in Hyades has already been investigated in detail by the author and his colleagues (Y1ld1z et al. 2006) and is therefore not included here. Thus, twelve DEBs in eleven open clusters are studied, namely V453 Cyg in NGC 6871, V1229 Tau (HD 23642) in Pleiades, V578 Mon in NGC 2244, V906 Sco in NGC 6475, V497 Cep in NGC 7160, V381 Car (HD 92024) in NGC 3293, V392 Car in NGC 2516, V1034 Sco in NGC 6231, DW Car in Cr 228, GV Car in NGC 3532, V615 Per and V618 Per in NGC 869. The masses of the binaries vary between $1.54 \mathrm{M}_{\odot}$ and $16.84 \mathrm{M}_{\odot}$; therefore, this study essentially deals with the structure and evolution of the early-type stars.

The key point for stellar evolution is the increase in mean molecular weight, which causes contraction in the nuclear core. The temperature of the core is increased by the heat released as a result of contraction, and hence nuclear reactions are intensified. This is the basic mechanism that controls the evolution and determines the timedependence of stellar structure. By constructing rotating (for the rapidly rotating components of DW Car) and nonrotating evolutionary models for the component stars in the DEBs, we obtain information about the age and chemical composition of the binaries and hence about their clusters. The information about age and chemical composition is deduced by fitting accurate values of luminosities and radii of component stars, if possible. We also obtain some simple expressions based on the slope of mass-radius and mass-luminosity relations for the binaries in order to find the age of binary systems easily. 
Table 1. The fundamental properties of the double-lined eclipsing binaries, which are members of open clusters

\begin{tabular}{|c|c|c|c|c|c|c|c|c|}
\hline Star & $M / M_{\odot}$ & $R / R_{\odot}$ & $\log \left(T_{\text {eff }}(\mathrm{K})\right)$ & $\log \left(L / L_{\odot}\right)$ & $t_{\mathrm{W}}(\mathrm{y})$ & $t_{\mathrm{Kh}}(\mathrm{y})$ & Cluster & Ref. \\
\hline V615 Per A & $4.075 \pm 0.055$ & $2.291 \pm 0.141$ & $4.176 \pm 0.033$ & $2.370 \pm 0.080$ & $1.172 \times 10^{7}$ & $1.91 \times 10^{7}$ & NGC869 & {$[1]^{a}$} \\
\hline V615 Per B & $179 \pm 0.051$ & $1.903 \pm 0.094$ & & & & & & \\
\hline V618 Per A & $332 \pm 0.031$ & $1.636 \pm 0.069$ & $4.041 \pm 0.033$ & $1.510 \pm 0.140$ & $1.172 \times 10^{7}$ & $1.91 \times 10^{7}$ & NGC869 & {$[1]^{a}$} \\
\hline V618 Per B & $558 \pm 0.025$ & $1.318 \pm 0.069$ & $3.903 \pm 0.045$ & $0.770 \pm 0.080$ & & & & \\
\hline V453 Cyg A & $14.36 \pm 0.20$ & $8.551 \pm 0.055$ & $4.424 \pm 0.019$ & $4.690 \pm 0.210$ & $9.08 \times 10^{6}$ & $9.77 \times 10^{6}$ & NGC6871 & {$[2]^{a}$} \\
\hline V453 Cyg B & $11.11 \pm 0.13$ & $5.489 \pm 0.063$ & $4.406 \pm 0.031$ & $4.240 \pm 0.280$ & & & & \\
\hline V1229 Tau A & $2.193 \pm 0.022$ & $1.831 \pm 0.029$ & $3.989 \pm 0.026$ & $1.437 \pm 0.047$ & $1.5 \times 10^{8}$ & $1.35 \times 10^{8}$ & Pleiades & [3] \\
\hline V1229 Tau B & $550 \pm 0.018$ & $1.548 \pm 0.0$ & 0.053 & $0.858 \pm$ & & & & \\
\hline V578 Mon A & $14.54 \pm 0.08$ & $5.23 \pm 0.06$ & $4.477 \pm 0.017$ & $4.291 \pm 0.188$ & $7.870 \times 10^{6}$ & $5.01 \times 10^{6}$ & NGC2244 & [4] \\
\hline V578 Mon B & $10.29 \pm 0.0$ & $4.32 \pm$ & .015 & $3.901=$ & & & & \\
\hline V906 Sco A & $3.253 \pm 0.069$ & $3.515 \pm 0.039$ & 0.047 & $2.162 \pm 0.082$ & $2.400 \times 10^{8}$ & $1.66 \times 10^{8}$ & NGC6475 & [5] \\
\hline V906 Sco B & $3.378 \pm$ & $4.521 \pm$ & & 2.33 & & & & \\
\hline V497 Cep A & $6.89 \pm 0.46$ & $3.69 \pm 0.03$ & $4.290 \pm$ & $3.265 \pm 0.144$ & $1.896 \times 10^{7}$ & $4.57 \times 10^{7}$ & NGC7160 & {$[6]^{a}$} \\
\hline V497 Cep B & $5.39 \pm 0.40$ & $2.92 \pm 0.03$ & $4.249 \pm$ & 2.860 & & & & \\
\hline V381 Car A & $15.0 \pm 3.0$ & $8.4 \pm 0.8$ & $4.406 \pm 0.020$ & $4.419 \pm 0.121$ & $1.032 \times 10^{7}$ & $8.71 \times 10^{6}$ & NGC3293 & [7] \\
\hline V381 Car B & $3.0 \pm 0.5$ & $2.1 \pm 0.4$ & & $1.980 \pm 0.290$ & & & & \\
\hline V392 Car A & $1.900 \pm 0.02$ & $1.625 \pm 0.030$ & $3.947 \pm 0.023$ & $1.162 \pm 0.055$ & $1.123 \times 10^{8}$ & $1.20 \times 10^{8}$ & NGC2516 & [8] \\
\hline V392 Car B & $1.853 \pm 0.02$ & $1.600 \pm 0.030$ & $3.937 \pm 0.023$ & $1.109 \pm 0.056$ & & & & \\
\hline V1034 Sco A & $16.84 \pm 0.48$ & $7.45 \pm 0.07$ & $4.471 \pm 0.015$ & $4.580 \pm 0.200$ & $6.967 \times 10^{6}$ & $6.46 \times 10^{6}$ & NGC6231 & [9] \\
\hline V1034 Sco B & $9.38 \pm 0.27$ & $4.18 \pm 0.04$ & $4.370 \pm 0.033$ & $3.672 \pm 0.210$ & & & & \\
\hline DW Car A & $11.34 \pm 0.12$ & $4.558 \pm 0.045$ & $4.446 \pm 0.016$ & $4.055 \pm 0.063$ & $6.761 \times 10^{6}$ & $4.79 \times 10^{6}$ & $\mathrm{Cr} 228$ & [10] \\
\hline DW Car B & $10.63 \pm 0.14$ & $4.297 \pm 0.055$ & $4.423 \pm 0.016$ & $3.915 \pm 0.067$ & & & & \\
\hline GV Car A & $2.51 \pm 0.03$ & $2.57 \pm 0.05$ & & $1.807 \pm 0.219$ & $3.105 \times 10^{8}$ & $2.82 \times 10^{8}$ & NGC3532 & [11] \\
\hline GV Car B & $1.54 \pm 0.02$ & $1.43 \pm 0.06$ & $3.903 \pm 0.045$ & $0.875 \pm 0.300$ & & & & \\
\hline
\end{tabular}

(1) Southworth et al. (2004a), (2) Southworth et al. (2004b), (3) Southworth et al. (2005), (4) Hensberge et al. (2000), (5) Alencar et al. (1997), (6) Yakut et al. (2003), (7) Freyhammer et al. (2005), (8) Debernardi \& North (2001), (9) Bouzid et al. (2005), (10) Southworth \& Clausen (2007), (11) Southworth \& Clausen (2006).

${ }^{a}$ The values given for the luminosities, radii and effective temperatures do not obey the luminosity equation $L=4 \pi R^{2} \sigma T_{\text {eff }}^{4}$ (see Section 3 and Table 3 ).

The ages of stars are derived by means of various astrophysical techniques including (i) observed surface properties (see, for example, Kharchenko et al. 2005; Yildız et al. 2006); (ii) seismic properties (in particular for the stars with the solar-like oscillations: see, for example, Yildız 2007, 2008; Eggenberger et al. 2008); (iii) rotational properties (Mamajek \& Hillenbrand 2008); and (iv) radioactive elements (del Peloso et al. 2006). The most widely used technique is based on the observed surface properties, which is also the method of the present study.

The Sun, with its precise seismic and non-seismic constraints, is the first target to model for an upgraded code describing stellar interiors. The agreement between the solar models and observed constraints gives an idea of the quality of the code. One can obtain the initial hydrogen $\left(X_{\mathrm{o}}\right)$ and heavy-element $\left(Z_{\mathrm{o}}\right)$ abundances and the mixing-length parameter of the Sun from the calibration of solar models, and use them when these quantities are not constrained for any star. In Yildiz (2008), $X_{\mathrm{o}}, Z_{\mathrm{o}}$ and $\alpha$ are found to be $0.70975,0.016$ and 1.82 , respectively, for the solar model with heavy element mixture of Asplund, Grevesse \& Sauval (2005) and with an age of $4.6 \mathrm{~Gy}$. The details of the code used in the model computations can also be found in Yıldız \& Kızıloğlu (1997) and Yildiz $(2007,2008)$.

The remainder of this paper is organized as follows. In Section 2, we present the observational fundamental properties of the DEBs in open clusters available in the literature. The models of their components are given in Section 3, and the methods of age determination based on the slopes of mass-radius and mass-luminosity relations are presented and discussed in Section 4. Error analysis for age is presented in Section 5. Finally, we list some concluding remarks in Section 6.

\section{Observational Data of the Binaries in Open Clusters}

Mass transfer obscures the dependence of the fundamental properties of component stars on secondary causes, such as time, chemical composition, rotation, and magnetic field. Therefore, semi-detached and contact binaries are beyond the scope of the present study. Accordingly, DEBs, member of open clusters, are studied. We have found in the literature studies of twelve DEBs in eleven open clusters. The observed properties of their component stars are listed in Table 1. The sixth and the seventh columns list the ages of the clusters given in the WEBDA database (http: / / obswww . unige.ch) and Kharchenko et al. (2005), respectively. For some of the systems, $L=4 \pi R^{2} \sigma T_{\text {eff }}^{4}$ is not satisfied (see Section 3 and Table 3). Figure 1 shows the observed HertzsprungRussell diagram (HRD) for the component stars of these eclipsing binaries. All the stars are either main-sequence (MS) stars or very close to the MS. The mass interval of the stars ranges from $1.54\left(\mathrm{GV}\right.$ Car B) to $16.84 \mathrm{M}_{\odot}$ 


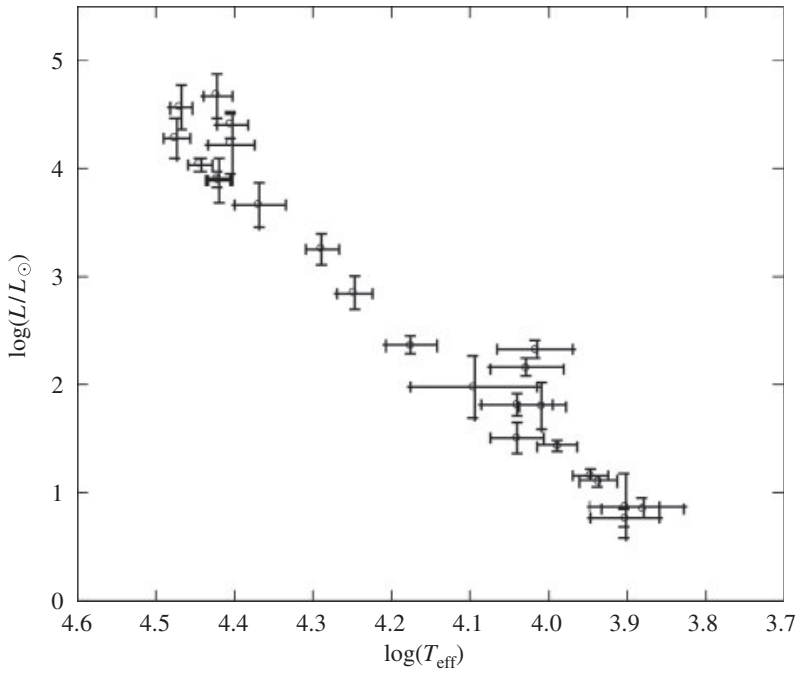

Figure 1 Observational HR diagram for the DEBs in open clusters.

(V1034 Sco A) and it covers a large part of the MS in the HRD from O-type to F-type stars. The ages of clusters vary between $5 \mathrm{My}$ and $310 \mathrm{My}$. In the upper part of the MS in Figure 1, from left to right, the ages of stars increase as theoretically expected. This result shows that the time dependence of effective temperature is more significant than any other second-order dependence (e.g. on chemical composition or rotation), at least for these stars.

The eleven clusters hosting the studied eclipsing binaries are at distances ranging from 150 (Pleiades) to 2327 pc (NGC3293). Number of stars in the clusters observed by UBV photometry is good enough to estimate the ages of the clusters by isochrone fitting. The WEBDA ages taken from different studies vary. Kharchenko et al. (2005) essentially use the isochrones of Girardi et al. (2002) for age determination.

These binaries have attracted attention in recent years. None of them is among the well-known binaries in Andersen (1991). However, six of them (V453 Cyg, V578 Mon, V906 Sco, V392 Car, V1034 Sco and DW Car) are listed in the recent compilation by Torres et al. (2010). Here we shall compare the basic properties of the stars in Table 1 with those given in Andersen (1991).

The slope of the mass-luminosity relation derived using the data in Table 1 is 3.89 . This means that

$$
L \propto M^{3.89}
$$

For the same mass range, we find $L \propto M^{4.02}$ for the DEBs with the most precise data on the fundamental properties (Andersen 1991). These two values for the power of $M$ are very close to each other. The slope of the mass-radius relation of the stars in Table 1 is found to be 0.704 . That is to say $R \propto M^{0.704}$. For the well-known eclipsing binaries (Andersen 1991), we find that $R \propto M^{0.82}$. The slopes we find for the mass-radius and mass-luminosity relations for binaries given in Table 1 are slightly less than the slopes found from the well-known binaries. The reason may be that many stars in the $\log \left(M / M_{\odot}\right)$ interval [0.1, 0.4] in Andersen (1991) have larger radii than their terminal-age MS (TAMS) radius. If we take the mass range as $\left[2.5 \mathrm{M}_{\odot}, 16.84 \mathrm{M}_{\odot}\right]$ rather than $\left[1.54 \mathrm{M}_{\odot}\right.$, $\left.16.84 \mathrm{M}_{\odot}\right]$, the slopes of the mass-luminosity and the mass-radius relations for the well-known binaries are found as 3.94 and 0.723 , respectively. These values are very close to the values we find for the eclipsing binaries in the open clusters.

\section{Ages of the Clusters from Models of the Component Stars in the Eclipsing Binaries}

The age of a binary system can be found from the solution of two equations based on the coevality of the component stars with two unknowns. One of the equations is written for the difference between the ages of the two components of the binary based on observed luminosities:

$$
\Delta t_{\mathrm{L}}=t_{\mathrm{LA}}-t_{\mathrm{LB}}
$$

where $t_{\mathrm{LA}}$ and $t_{\mathrm{LB}}$ are ages found from the luminosities of the primary and secondary components, respectively. The other equation based on the radii:

$$
\Delta t_{\mathrm{R}}=t_{\mathrm{RA}}-t_{\mathrm{RB}}
$$

where $t_{\mathrm{RA}}$ and $t_{\mathrm{RB}}$ are ages from the radii of the primary and secondary components, respectively. From the assumption of coevality, $\Delta t_{\mathrm{L}}=\Delta t_{\mathrm{R}}=0$. Using numerical derivatives of $\Delta t_{\mathrm{L}}$ and $\Delta t_{\mathrm{R}}$ with $X$ and $Z$, we solve these equations and find $X$ and $Z$. In general, we find that different combinations of $X$ and $Z$ for a given binary give similar results; thus, the solution is not unique. If it is not possible to obtain an acceptable solution to Eqs. (2) and (3) for a binary system, then we either use one of these equations or fit the model of one of its components to observed values.

In the following subsections, interior models of the component stars in Table 1 constructed using the stellar evolution code described in Y1ldız \& Kızıloğlu (1997) and Yildiz $(2007,2008)$ are presented. From these models, the ages and chemical compositions of the binaries are obtained if possible.

\subsection{On the Fundamental Properties of Components of V615 Per and V618 Per}

In this subsection, we consider the binary systems V615 Per and V618 Per in more detail. Their components give very different ages. The reason for this difference should be investigated. For V615 Per, we compare its components with those of V618 Per and find that the luminosity of V615 Per B is lower than is expected for its mass. If we assume that the power of mass is 4 for the slope of the mass-luminosity relation, then $\log \left(L_{B} / L_{\odot}\right)=2.02$, which is larger than the value found from observations with by amount of 0.2 . Since the slope of the mass-radius relation for the components of V615 Per and V618 
Table 2. The chemical composition and age of the binary systems derived from the calibration of models. The ages we find are listed in the fourth column. For comparison, the ages of the clusters given in the WEBDA database and by Kharchenko et al. (2005) are also presented. The seventh column reports the MS life time of the primary components of the binary systems. In the eighth column, the age found from the ratio of radii of the component stars is shown (see Section 4). The ages found from the fitting formula given in Eqs. (6-9) are listed in the ninth column

\begin{tabular}{|c|c|c|c|c|c|c|c|c|c|}
\hline Star & $X$ & $Z$ & $t(\mathrm{y})$ & $t_{\mathrm{W}}(\mathrm{y})$ & $t_{\mathrm{Kh}}(\mathrm{y})$ & $t_{\mathrm{MSA}}$ & $t_{\mathrm{rm}}$ & $t_{\mathrm{rmf}}$ & Cluster \\
\hline V615 Per & 0.7350 & 0.0100 & $2 \times 10^{7}$ & $1.172 \times 10^{7}$ & $1.91 \times 10^{7}$ & $1.40 \times 10^{8}$ & $2.92 \times 10^{7}$ & $2.71 \times 10^{7}$ & NGC 869 \\
\hline V618 Per & 0.7350 & 0.0100 & $2 \times 10^{7}$ & $1.172 \times 10^{7}$ & $1.91 \times 10^{7}$ & $5.98 \times 10^{8}$ & - & - & NGC 869 \\
\hline V453 Cyg & 0.7046 & 0.0117 & $1.05 \times 10^{7}$ & $9.08 \times 10^{6}$ & $9.77 \times 10^{6}$ & $1.18 \times 10^{7}$ & $9.76 \times 10^{6}$ & $9.60 \times 10^{6}$ & NGC 6871 \\
\hline V1229 Tau & 0.6946 & 0.0158 & $1.93 \times 10^{8}$ & $1.5 \times 10^{8}$ & $1.35 \times 10^{8}$ & $6.88 \times 10^{8}$ & $1.24 \times 10^{8}$ & $1.44 \times 10^{8}$ & Pleiades \\
\hline V578 Mon & 0.7098 & 0.0160 & $3.50 \times 10^{6}$ & $7.870 \times 10^{6}$ & $5.01 \times 10^{6}$ & $1.29 \times 10^{7}$ & - & - & NGC 2244 \\
\hline V906 Sco & 0.7200 & 0.0170 & $2.52 \times 10^{8}$ & $2.400 \times 10^{8}$ & $1.66 \times 10^{8}$ & $2.53 \times 10^{8}$ & $2.53 \times 10^{8}$ & $2.53 \times 10^{8}$ & NGC 6475 \\
\hline V497 Cep & 0.7440 & 0.0125 & $2.30 \times 10^{7}$ & $1.896 \times 10^{7}$ & $4.57 \times 10^{7}$ & $4.95 \times 10^{7}$ & $2.17 \times 10^{7}$ & $2.03 \times 10^{7}$ & NGC 7160 \\
\hline V381 Car & 0.7380 & 0.0258 & $1.05 \times 10^{7}$ & $1.032 \times 10^{7}$ & $8.71 \times 10^{6}$ & $1.37 \times 10^{7}$ & $1.05 \times 10^{7}$ & $0.43 \times 10^{7}$ & NGC 3293 \\
\hline V392 Car & 0.6837 & 0.0178 & $1.23 \times 10^{8}$ & $1.123 \times 10^{8}$ & $1.20 \times 10^{8}$ & $1.04 \times 10^{9}$ & $1.41 \times 10^{8}$ & $3.01 \times 10^{8}$ & NGC 2516 \\
\hline V1034 Sco & 0.6961 & 0.0226 & $5.51 \times 10^{6}$ & $6.967 \times 10^{6}$ & $6.46 \times 10^{6}$ & $1.02 \times 10^{6}$ & $5.36 \times 10^{6}$ & $4.21 \times 10^{6}$ & NGC 6231 \\
\hline DW Car & 0.6934 & 0.0106 & $5.22 \times 10^{6}$ & $6.761 \times 10^{6}$ & $4.79 \times 10^{6}$ & $1.56 \times 10^{7}$ & $4.88 \times 10^{6}$ & $5.72 \times 10^{6}$ & $\mathrm{Cr} 228$ \\
\hline$"$ (Rotat.) & 0.6951 & 0.0078 & $5.29 \times 10^{6}$ & $6.761 \times 10^{6}$ & $4.79 \times 10^{6}$ & $1.52 \times 10^{7}$ & $4.75 \times 10^{6}$ & $5.75 \times 10^{6}$ & $\operatorname{Cr} 228$ \\
\hline GV Car & 0.7046 & 0.0104 & $3.22 \times 10^{8}$ & $3.105 \times 10^{8}$ & $2.82 \times 10^{8}$ & $4.22 \times 10^{8}$ & $3.21 \times 10^{8}$ & $3.04 \times 10^{8}$ & NGC 3532 \\
\hline
\end{tabular}

Table 3. The modifications in the fundamental properties of the double-lined eclipsing binaries due to inconsistent results given in the literature are presented in bold

\begin{tabular}{lccc}
\hline Star & $M / M_{\odot}$ & $R / R_{\odot}$ & $\log \left(T_{\text {eff }}(\mathrm{K})\right)$ \\
\hline V615 Per A & $4.075 \pm 0.055$ & $2.291 \pm 0.141$ & $4.176 \pm 0.033$ \\
V615 Per B & $3.179 \pm 0.051$ & $1.903 \pm 0.094$ & $\mathbf{4 . 0 7 9} \pm 0.045$ \\
V615 Per A & $4.075 \pm 0.055$ & $2.291 \pm 0.141$ & $4.176 \pm 0.033$ \\
V615 Per B & $3.179 \pm 0.051$ & $1.903 \pm 0.094$ & $\mathbf{4 . 1 1 4} \pm 0.045$ \\
V618 Per A & $2.332 \pm 0.031$ & $1.636 \pm 0.069$ & $4.041 \pm 0.033$ \\
V618 Per B & $1.558 \pm 0.025$ & $1.318 \pm 0.069$ & $3.903 \pm 0.045$ \\
V453 Cyg A & $14.36 \pm 0.20$ & $8.551 \pm 0.055$ & $4.424 \pm 0.019$ \\
V453 Cyg B & $11.11 \pm 0.13$ & $5.489 \pm 0.063$ & $4.406 \pm 0.031$ \\
V497 Cep A & $6.89 \pm 0.46$ & $3.69 \pm 0.03$ & $4.290 \pm 0.021$ \\
V497 Cep B & $5.39 \pm 0.40$ & $2.92 \pm 0.03$ & $4.249 \pm 0.023$ \\
\hline
\end{tabular}

Per seems reasonable, we deduce that the effective temperature of V615 Per B should be larger than the value found by Southworth, Maxted \& Smalley (2004a). As a matter of fact, the results they give for the effective temperatures of V615 Per B $\left(M_{\mathrm{B}}=3.18 \pm 0.05 \mathrm{M}_{\odot}\right)$ and $\mathrm{V} 618 \operatorname{Per} \mathrm{A}\left(M_{\mathrm{A}}=2.33 \pm 0.03 \mathrm{M}_{\odot}\right)$ are the same, despite the very different masses.

Reasonable models for the components of V618 Per are obtained with $Z=0.01$ and $X=0.735$. In the HRD, the positions of the models at the ages of the cluster given in WEBDA and by Kharchenko et al. are the same for V618 Per A. For V618 Per B, the age =19.1 My (Kharchenko et al. 2005) is more suitable than $11.7 \mathrm{My}$ (WEBDA). At 11.7 My, V618 Per B is in the pre-MS stage. The ages and the chemical compositions we find are listed in Table 2.

The accurate values of the components of V615 and V618 Per given in Southworth (2004a) do not exactly obey $L=4 \pi R^{2} \sigma T_{\text {eff. According to Southworth (private }}^{4}$ communication), this arises because the same distance for the two components of a DEB is enforced in the lightcurve analysis. For the system V615 Per, however, the problem is with V615 Per B, particularly with its effective temperature. For its $\log \left(L / L_{\odot}\right)=1.82$ and $R / R_{\odot}=1.903$ values, its effective temperature should be $12,000 \mathrm{~K}$ rather than $11,000 \mathrm{~K}$. The suggested values are given in bold in Table 3 .

For age determination of these systems, we use observational radii of the component stars as constraints for their interior models, because of the complicated luminosities and effective temperatures. The age of these systems is roughly estimated as $20 \mathrm{My}$. V615 Per B seems to be a MS star at this age.

\subsection{On the Fundamental Properties of Components of $V 906 \mathrm{Sco}$}

The difference between the masses of the components of V906 Sco is not very large but the primary component is a TAMS star and therefore very appropriate for age determination. The ages are found from the solution of Eqs. (2) and (3), based on the coevality of the component stars. We solve these equations and find $X$ and $Z$. Using different reference models, we obtain very different combinations of $X$ and $Z$, and hence the solution is not unique. However, the ages found from the luminosities and radii of V906 Sco A and B are very close to each other. For the three cases given in Table 4 , the age found from $R_{\mathrm{B}}$ (the last column) is slightly less than the others. The mean value of ages is $252 \mathrm{My}$. Without $t_{\mathrm{RB}}$ it is $256 \mathrm{My}$. 
Table 4. Ages derived from fitting the luminosities $\left(t_{\mathrm{L}}\right)$ and radii $\left(t_{R}\right)$ of models for V906 Sco A and B to the observed values, for different combinations of hydrogen and heavy element abundances. While the mean of all values is $252 \mathrm{My}$, the mean value of $t_{\mathrm{LA}}, t_{\mathrm{LB}}$ and $t_{\mathrm{RA}}$ is $256 \mathrm{My}$

\begin{tabular}{lccccc}
\hline$X$ & $Z$ & $t_{\mathrm{LA}}(\mathrm{My})$ & $t_{\mathrm{LB}}(\mathrm{My})$ & $t_{\mathrm{RA}}(\mathrm{My})$ & $t_{\mathrm{RB}}(\mathrm{My})$ \\
\hline 0.720 & 0.0170 & 256 & 256 & 253 & 235 \\
0.729 & 0.0156 & 256 & 259 & 258 & 240 \\
0.737 & 0.0142 & 257 & 260 & 253 & 244 \\
\hline
\end{tabular}

\subsection{V578 Mon}

This system has the lowest values for slopes of the massluminosity and mass-radius relations among the systems withthe early-type components:

$$
\begin{aligned}
& l_{m}=\log \left(L_{\mathrm{A}} / L_{\mathrm{B}}\right) / \log \left(M_{\mathrm{A}} / M_{\mathrm{B}}\right)=2.6, \\
& r_{m}=\log \left(R_{\mathrm{A}} / R_{\mathrm{B}}\right) / \log \left(M_{\mathrm{A}} / M_{\mathrm{B}}\right)=0.55 .
\end{aligned}
$$

This means that either the observational values of $L_{\mathrm{A}}$ and $R_{\mathrm{A}}$ are very low or the the observational values of $L_{\mathrm{B}}$ and $R_{\mathrm{B}}$ are very high, compared to the values expected from their masses. The influence of this point on the age determination of the system is crucial.

Abundances of some heavy elements (including C, N and $\mathrm{O}$ ) are found from disentangled spectra by Pavlovski and Hensberge (2005). The oxygen is underabundant by about -0.10 dex. If we take the solar $Z_{s}=0.0122$ (surface heavy element abundance), then $Z_{0}$ of NGC 2244 is about 0.01 .

From the models of the component stars with solar composition $\left(X_{\mathrm{o}}=0.70975, Z_{\mathrm{o}}=0.016\right)$, we find the agreement time for the quantities $L_{\mathrm{A}}, L_{\mathrm{B}}, R_{\mathrm{A}}$ and $R_{\mathrm{B}}$. While $t_{\mathrm{LA}}$ and $t_{\mathrm{RA}}$ are about $3.5 \mathrm{My}, t_{\mathrm{LB}}$ and $t_{\mathrm{RB}}$ are about three and two times larger than $3.5 \mathrm{My}$, respectively. The reason for the small radius of V578 Mon A or the large radius of V578 Mon B may be the accuracy of the data. However, if this is not the case then this system has some interesting features which are beyond the standard models, for example, the existence of a rapidly rotating core for V578 Mon A (Y1ld1z 2003, 2005) or rotational mixing (Meynet \& Maeder 2000). Within the standard approach, the present data implies that V578 Mon B is more evolved than V578 Mon A. The compatible radius for V578 Mon A based on its mass is about $6 R_{\odot}$, which is $15 \%$ larger than the observed value. Assuming the same effective temperature as given by Hensberge et al. (2000), the increase in luminosity of V578 Mon A due to the increase in its radius is about $30 \%$. For this radius and luminosity, the age of the system is found as $6 \mathrm{My}$ from the models of V578 Mon A and B with $Z=0.01$ and $X=0.710$.

Hensberge et al. (2000) show that the position of V578 Mon A in HRD corresponds to $11 M_{\odot}$. This may be a manifestation of the rapidly rotating cores of early-type stars

\subsection{V392 Car}

The masses of the components of V392 Car are very close to each other: $M_{\mathrm{A}}=1.90 \pm 0.02 \mathrm{M}_{\odot}, M_{\mathrm{B}}=1.853 \pm 0.02 \mathrm{M}_{\odot}$
Table 5. Ages derived from fitting the luminosities $\left(t_{\mathrm{L}}\right)$ and radii $\left(t_{R}\right)$ of models for $V 392$ Car $A$ and $B$ to the observed values, for different combinations of hydrogen and heavy element abundances

\begin{tabular}{lccccc}
\hline$X$ & $Z$ & $t_{\mathrm{LA}}(\mathrm{My})$ & $t_{\mathrm{LB}}(\mathrm{My})$ & $t_{\mathrm{RA}}(\mathrm{My})$ & $t_{\mathrm{RB}}(\mathrm{My})$ \\
\hline 0.7200 & 0.0135 & 260 & 200 & 273 & 284 \\
0.7020 & 0.0155 & 200 & 156 & 189 & 203 \\
0.6900 & 0.0174 & 220 & 182 & 140 & 156 \\
0.6837 & 0.0178 & 157 & 98 & 126 & 122 \\
0.6825 & 0.0180 & 163 & 101 & 119 & 116 \\
0.6880 & 0.0184 & 246 & 182 & 112 & 105 \\
0.6800 & 0.0184 & 165 & 99 & 111 & 102 \\
\hline
\end{tabular}

(Debernardi \& North 2001). In Table 5, the ages found from the luminosities and radii for different combinations of $X$ and $Z$ are listed. For such systems with similar components, small differences due to uncertainties in the observed quantities may lead to very different results in model computations. However, the observed values of the radii of the component stars are very accurate and provide an opportunity to determine the age of the system very accurately. The models with $X=0.6837$ and $Z=0.0178$ predict very close ages for V392 Car A and B (126 and 122 My, respectively). The adopted value for the age, $124 \mathrm{My}$, is the mean of these values. Debernardi \& North (2001) reported very similar metallicity for V392 Car, $Z=0.018$, using the evolutionary tracks of Schaller et al. (1992).

\subsection{V1229 Tau (HD 23642)}

The fundamental properties of the components of V1229 Tau are determined from light and radial velocity curves by Munari et al. (2004), Southworth et al. (2005) and Groenewegen et al. (2007). The results of these studies are very similar. Among the eclipsing binaries in open clusters considered, this system has the minimum value of $r_{m}=\log \left(R_{\mathrm{A}} / R_{\mathrm{B}}\right) / \log \left(M_{\mathrm{A}} / M_{\mathrm{B}}\right)$.

It is not possible to fit models of V1229 Tau A and B to the observed properties simultaneously. Therefore, we find the age of the system by considering V1229 Tau A and B separately.

The age derived from the calibration of V1229 Tau A is 193 My for $(X, Z)=(0.6946,0.01575)$ and $222 \mathrm{My}$ for $(X, Z)=(0.7104,0.014)$. However, the age derived from the calibration of V1229 Tau B is $730 \mathrm{My}$ for $(X, Z)=$ $(0.7046,0.01305)$. This value is about five times greater than the value given by Kharchenko et al. In comparison to the age found for V1229 Tau B, the ages found for V1229 Tau A are close to the ages of Kharchenko et al. Southworth et al. (2005) find an age about 125-175 My, close to the results from the calibration of V1229 Tau A. The age of V1229 Tau is listed as 193 My in Table 2.

\subsection{V453 Cyg}

Recently, precise fundamental properties of V453 Cyg A and B have been presented by Southworth, Maxted \& Smalley (2004b). However, there is a contradiction in their results. According to the radii and effective 
temperatures of the components, the luminosities must be lower than the published values; in their Table 11, they give $\log \left(L_{\mathrm{A}} / L_{\odot}\right)=4.69$ and $\log \left(L_{\mathrm{B}} / L_{\odot}\right)=4.24$ but we compute the luminosities from the radii and effective temperatures given in that table and find that $\log \left(L_{\mathrm{A}} / L_{\odot}\right)=4.513$ and $\log \left(L_{\mathrm{B}} / L_{\odot}\right)=4.056$. The correction in $\log (L)$ is about 0.18 or $50 \%$ (see Table 3 ).

From the calibration of model radii of V453 Cyg A and B for $X=0.700$, we find the age of the system as $10.3 \mathrm{My}$ and $Z=0.0174$. For $X=0.7046$, however, the age and $Z$ are computed as $10.5 \mathrm{My}$ and 0.0117 , respectively. Whereas the ages found with very different chemical compositions are very close, the model luminosities with the latter values are in perfect agreement with the (corrected) values found from observations. Southworth, Maxted \& Smalley (2004b) give the age of the system as $10 \mathrm{My}$. The ages determined for V453 Cyg are in good agreement with this age and the age of the cluster given by Kharchenko et al. (2005). Such a good agreement is based on the fact that V453 Cyg A is very close to the TAMS.

Abundance analysis of V453 Cyg A and B is made by Pavlovski \& Southworth (2009). They found that N, O and Mg abundances for V453 Cyg A are nearly solar and $\mathrm{C}, \mathrm{Si}$ and $\mathrm{Al}$ are underabundant. Regarding the fact that $\mathrm{O}$ is the most abundant heavy element for normal stars, we estimate that $Z \simeq 0.01$ from oxygen abundance ( -0.08 dex) estimated by Pavlovski \& Southworth (2009). This value is in good agreement with the values predicted from the models $(Z=0.0117)$. Pavlovski \& Southworth also re-derived the effective temperatures of V453 Cyg $\mathrm{A}$ and B: $T_{\text {eff A }}=27,900 \pm 400 \mathrm{~K}$ and $T_{\text {eff B }}=26,200 \pm$ $500 \mathrm{~K}$. These values are 5 and $3 \%$ greater than the values given by Southworth, Maxted \& Smalley (2004b).

Pavlovski \& Southworth forecast the helium abundance as 0.086 and $0.123 \mathrm{dex}$, depending upon the method for derivation of microturbulence velocity. For these helium abundances, very similar ages are found: for $0.086 \mathrm{dex}, Z=0.0113$ and the mean age is $10 \mathrm{My}$; for $0.123 \mathrm{dex}, Z=0.0163$ and the mean age is $9 \mathrm{My}$.

\subsection{V497 Cep}

The fundamental properties of this system were obtained by Yakut et al. (2003). Using their findings, the best agreement between the models and the observational results from photometric and spectroscopic analysis is reached with $X=0.744$ and $Z=0.0125$. The derived age of $23.0 \mathrm{My}$ is the average of $t_{\mathrm{LA}}=t_{\mathrm{RA}}=22.3 \mathrm{My}$, $t_{\mathrm{LB}}=24.9 \mathrm{My}$ and $t_{\mathrm{RB}}=22.5 \mathrm{My}$. Whereas the age we find is close to the age for the cluster given in WEBDA, it is half of the value derived by Kharchenko et al. (2005).

The accurate values given in Yakut et al. (2003) do not exactly obey $L=4 \pi R^{2} \sigma T_{\text {eff. }}^{4}$ It is not easy to find the source of this inconsistency. However, it may be due to rounding off.

\subsection{V381 Car (HD 92024)}

V381 Car A is a $\beta$ Cep for which three oscillation frequencies are observed with $l=2$ and 4 (Freyhammer et al. 2006). Despite the large uncertainty in the fundamental properties of its components (Freyhammer et al. $2005)$, it is a normal system with its slopes for the massradius and mass-luminosity relations given as 0.861 and 3.4894. From the fit of model radii and luminosities to the observed values, with $X=0.738$ and $Z=0.0258$, we find that $t_{\mathrm{LA}}=t_{\mathrm{RA}}=10.8 \mathrm{My}$ and $t_{\mathrm{RB}}=9.8 \mathrm{My}$. The age of the system is found as $10.5 \mathrm{My}$, the average of these values. This age is in agreement with the age range (10-13 My) estimated by Freyhammer et al. (2005).

The chemical composition found from fitting interior models of V381 Car A and B is very different from that of the other binaries in the sense that both $X$ and $Z$ are greater than the solar values. This may be a result of the overestimated masses of V381 Car A and B.

\subsection{V1034 Sco}

It is a normal system, with slopes for the mass-radius and mass-luminosity relations of 0.9876 and 3.5728 , respectively. From the fit of model radii and luminosities to the observed values, with $X=0.6961$ and $Z=0.02255$, we find that $t_{\mathrm{RA}}=t_{\mathrm{RB}}=5.64 \mathrm{My}, t_{\mathrm{LA}}=5.72 \mathrm{My}$ and $t_{\mathrm{LB}}=5.05 \mathrm{My}$. The age of the system is computed as $5.51 \mathrm{My}$ by taking the average of these values. Bouzid et al. (2005) confirm that the age of the system is about $5 \mathrm{My}$, in good agreement with the findings of the present study.

\section{$3.10 \mathrm{DW} C a r$}

At first sight, this system seems a normal system with slopes for the mass-radius and mass-luminosity relations of 0.9120 and 4.9858, respectively (Southworth \& Clausen 2007). According to the radii, the components of DW Car are unevolved MS stars and very close the zero-age MS (ZAMS). However, the luminosity derived from the observation implies that DW Car A is an evolved MS star. No simultaneous solution is available for the luminosities of DW Car A and B. From their radii, the age of the system is calculated as $5.22 \mathrm{My}$. The non-rotating models giving this age are constructed with $X=0.6934$ and $Z=0.01063$. However, DW Car A and B are fast rotators; $v_{\mathrm{eq} \mathrm{A}}=182 \pm 3 \mathrm{~km} \mathrm{~s}^{-1}$ and $v_{\mathrm{eq} \mathrm{B}}=177 \pm 3$ $\mathrm{km} \mathrm{s}^{-1}$. Therefore, models rotating like a solid body are also constructed for them. The calibration of rotating models results in nearly the same age, 5.31 My. The hydrogen and metal abundances for the rotating models are $X=0.6951$ and $Z=0.0078$, very close to that of nonrotating models. Southworth \& Clausen (2007) reported similar results: about $6 \mathrm{My}, Z \simeq 0.01$.

\subsection{GV Car}

This system consists of two metallic-line stars and it shows apsidal motion. From the calibration of models of the components, with $X=0.7046$ and $Z=0.0104$, we estimate that $t_{\mathrm{RA}}=324 \mathrm{My}, t_{\mathrm{RB}}=372 \mathrm{My}, t_{\mathrm{LA}}=295 \mathrm{My}$ and $t_{\mathrm{LB}}=302 \mathrm{My}$. The age of the system is found as $322 \mathrm{My}$. Southworth \& Clausen (2006) give a very similar age for GV Car, $360 \mathrm{My}$. 
Table 6. Luminosity and radius difference between ZAMS and TAMS for evolutionary tracks of V906 Sco A. $\Delta \log (L)=\left(L_{\mathrm{TAMS}}-L_{\mathrm{ZAMS}}\right) / L_{\mathrm{TAMS}}, \Delta \log (R)=\left(R_{\mathrm{TAMS}}-R_{\mathrm{ZAMS}}\right) /$ $R_{\text {TAMS }}$

\begin{tabular}{lcccc}
\hline Star & $X$ & $Z$ & $\Delta \log (L)$ & $\Delta \log (R)$ \\
\hline V906 Sco A & 0.702 & 0.0210 & 0.458 & 0.439 \\
& 0.720 & 0.0170 & 0.456 & 0.444 \\
& 0.729 & 0.0156 & 0.457 & 0.448 \\
GV Car A & 0.737 & 0.0142 & 0.456 & 0.446 \\
& 0.705 & 0.0200 & 0.431 & 0.422 \\
& 0.720 & 0.0100 & 0.442 & 0.423 \\
\hline
\end{tabular}

\section{Mass and Time Dependence of the Mass-Radius and Mass-Luminosity Relations}

The luminosity and radius of a non-rotating star are functions of its mass, chemical composition ( $X$ and $Z$ ), and age: $L=L(M, X, Z, t), R=R(M, X, Z, t)$.

Changes in $X$ and $Z$ shift the evolutionary track of a model for a given mass in HRD. Despite the variation in the positions of ZAMS and TAMS points according to chosen values of $X$ and $Z$, the ratio of TAMS to ZAMS luminosity is independent of $X, Z$ and stellar mass: the logarithmic luminosity difference, $\Delta \log (L)=L_{\mathrm{TAMS}}-$ $\left.L_{\text {ZAMS }}\right) / L_{\text {TAMS }}$, is constant. In Table $6, \Delta \log (L)$ and $\Delta \log (R)$ for the models of V906 Sco A and GV Car A are given in the fourth and the fifth columns, respectively. For very different combinations of $X$ and $Z$, the differences are between 0.42 and 0.46 : the ZAMS luminosity (radius) is about $44 \%$ less than the TAMS luminosity (radius), at least for the early-type stars. Consider a binary system whose component masses are very different, so that the massive component is near the TAMS and the low-mass component is still near the ZAMS. For such a system, the luminosity ratio $\left(L_{\mathrm{A}} / L_{\mathrm{B}}\right)$ is at a maximum. It was at a minimum when both of the components were near the ZAMS, and gradually increased with time. This is the case also for the ratio of the radii of the components. In this section, we discuss variations in the radius and luminosity ratios with time and then develop two methods for age determination of eclipsing binaries.

As stated above, the mass-luminosity relation is not constant and mainly depends on the stellar mass. It increases with time. In Figure 2, the luminosity-mass ratio $\left(l_{m}=\log \left(L_{\mathrm{A}} / L_{\mathrm{B}}\right) / \log \left(M_{\mathrm{A}} / M_{\mathrm{B}}\right)\right)$ obtained from the models with values given in Table 2 is plotted with respect to the relative age in MS $\left(t / t_{\mathrm{MSA}}\right)$. Whereas $l_{m}$ increases with time, there is an inverse relation between $l_{m}$ and $M_{\mathrm{A}}$. Near the ZAMS, $l_{m}$ varies between 3 and 4 and takes values between 4 and 5 near the TAMS. In principle, $l_{m}$ is very useful for predicting the age of a system if its accuracy is good enough.

The MS lifetime of the primary stars $t_{\mathrm{MSA}}$, given in the seventh column of Table 2, is mainly a function of $M_{\mathrm{A}}$, despite very different chemical compositions found for the DEBs. From a fit to the $M_{\mathrm{A}}-t_{\mathrm{MSA}}$ relation we find that

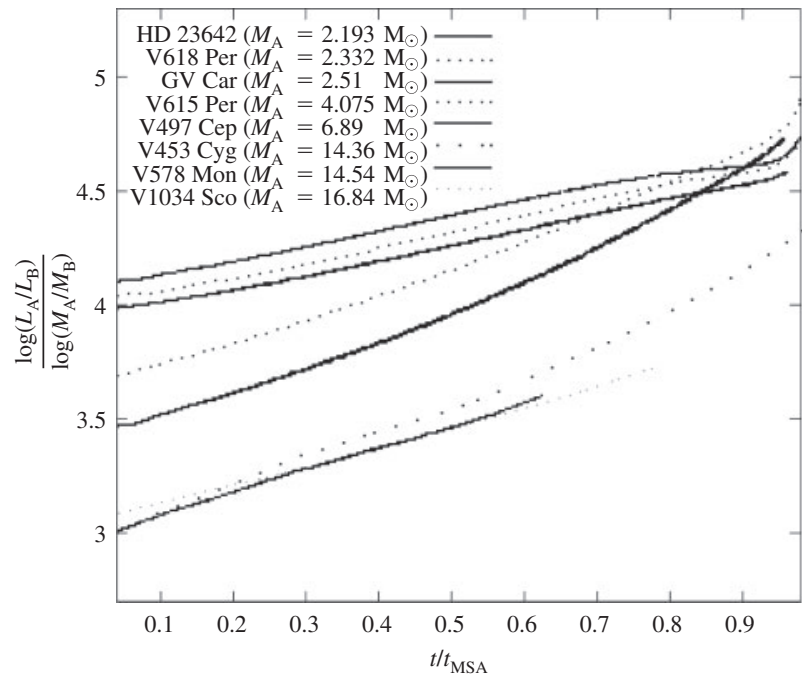

Figure 2 The mass-luminosity relation for the DEBs in open clusters is plotted with respect to the relative age of the primary components.

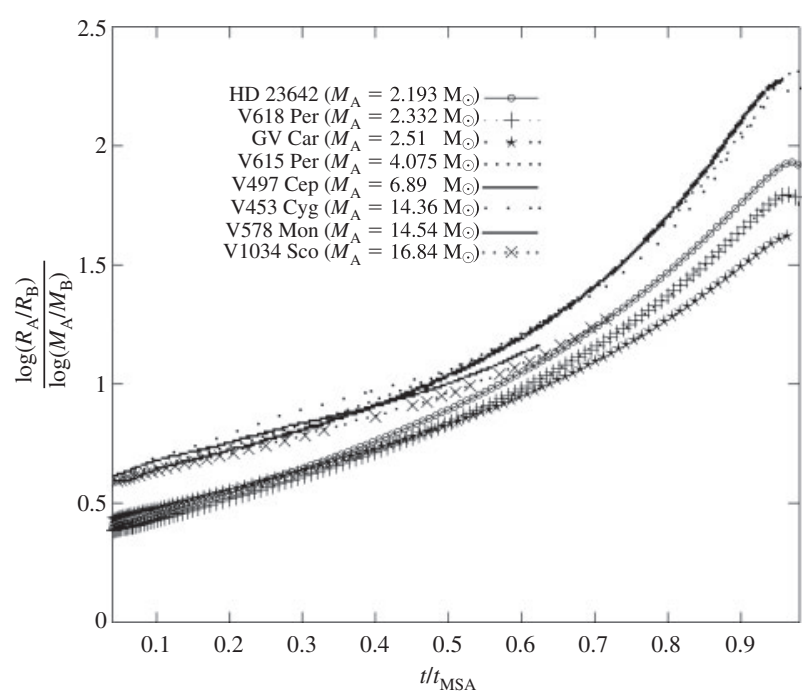

Figure 3 The mass-radius relation for the DEBs in open clusters.

$$
t_{\mathrm{MS}}=\frac{3.37 \times 10^{9}}{\left(M / M_{\odot}\right)^{2.122}}
$$

\subsection{Age Determination from the Slope of the Mass-Radius Relation}

The slope of the mass-radius relation $r_{m}=\log \left(R_{\mathrm{A}} / R_{\mathrm{B}}\right) /$ $\log \left(M_{\mathrm{A}} / M_{\mathrm{B}}\right)$ is also a function of the primary mass and time. In Figure $3, r_{m}$ is plotted with respect to $t / t_{\mathrm{MSA}}$. From the curves in Figure 3, we determine the relative age of the binary systems by using the observed values of $r_{m}$. Multiplication of the relative age of the primary component of a binary system by $t_{\mathrm{MSA}}$ gives us age of that binary. The ages of all the binaries computed with this method $\left(t_{\mathrm{rm}}\right)$ are listed in the eighth column of Table 2. In Figure 4, these ages (filled circles) are plotted with respect to the ages from fitting interior models of component stars to the 


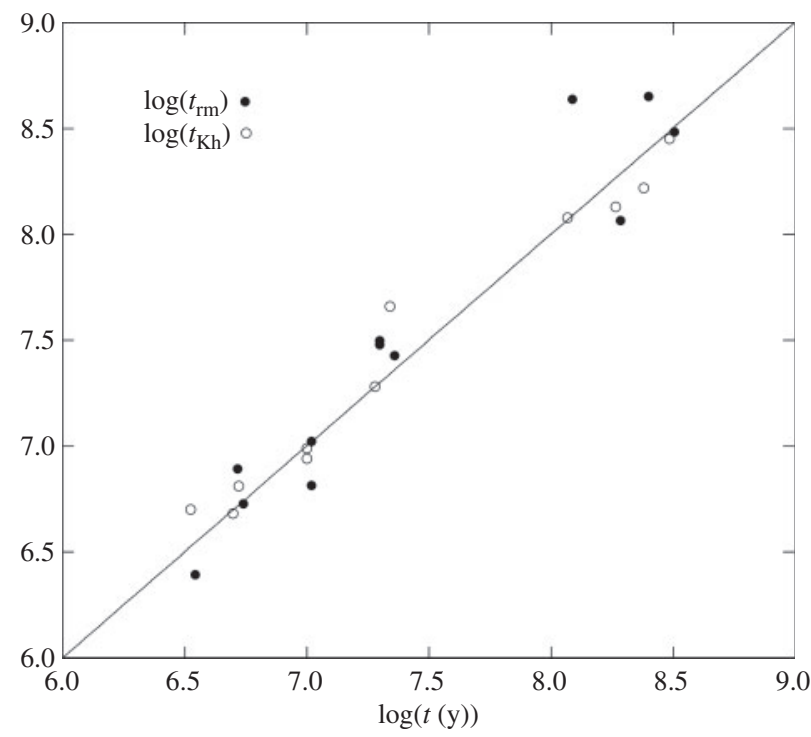

Figure 4 The ages of binaries derived from the ratio of radii (filled circle) are plotted with respect to the ages found from fitting models. For comparison, ages given by Kharchenko et al. (2005) are also shown (circle).

observed accurate values (the fifth column in Table 2). For comparison, the ages found by Kharchenko et al. (2005) are also shown (circle). There is in general a good agreement between the ages, except for V618 Per and V578 Mon. The observed value of $r_{m}$ is very high for V618 Per and low for V578 Mon, in comparison with the model values. For V578 Mon, Garcia et al. (2010) report that they find a different value for the secondary radius. The difference may significantly influence the observed value of $r_{m}$.

The age of V1229 Tau is computed as 124 My by using $r_{m}$ from Munari et al. (2004). $r_{m}$ from Southworth et al. (2005) gives the age of the system as $97 \mathrm{My}$.

The precision of the ages found from the mass-radius curves depends on how sensitive the relative age is to chemical composition. In Figure $5, r_{m}$ values derived from models of V618 Per A and B with different chemical compositions are plotted with respect to the relative age $t / t_{\mathrm{MSA}}$. For $X=0.705$, there is a perfect agreement between $r_{m}$ values of models with $Z=0.01$ (thin solid line) and $Z=0.02$ (dotted line). We shall also compare $r_{m}$ values of models with the same $Z$ but different $X$. The $r_{m}$ of models with $X=0.735$ and $Z=0.01$ (thick solid line) is slightly less than that of the models with $X=0.705$ and $Z=0.01$. The agreement between the three curves of $r_{m}$ is good enough to suggest that relation between $r_{m}$ and relative age is almost independent of chemical composition. For the precision of the ages, the sensitivity of $t_{\mathrm{MSA}}$ to chemical composition is also important. As given in Eq. (4), $t_{\mathrm{MSA}}$ is a very strong function of stellar mass. The secondary effect of $Z$ on $t_{\mathrm{MSA}}$ behaves as $t_{\mathrm{MSA}} \propto(Z / 0.015)^{0.43}$ and is neglected in the present study.

\subsection{Simple Expressions for the $\mathrm{r}_{\mathrm{m}}$-Age Relation}

The time dependence of $r_{m}$ of binary systems with very different masses and chemical compositions is very

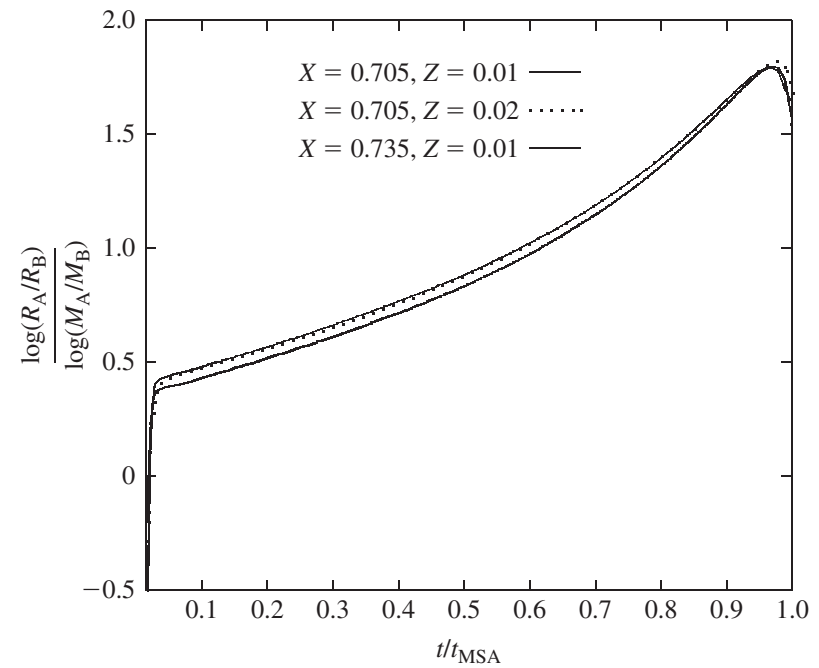

Figure 5 The mass-radius relation for models of V618 Per with different $X$ and $Z$.

similar and therefore can be used to estimate the age of a binary system. To do this, the time variation of $r_{m}$ is fitted with an equation of the form

$$
r_{m}=a\left(t / t_{\mathrm{MSA}}\right)^{n}+b .
$$

According to $M_{\mathrm{A}}$ of the binaries, the curves of $r_{m}$ in Figure 3 separate essentially into two groups. The $r_{m}$ values of the binaries with $M_{\mathrm{A}}<3.4 \mathrm{M}_{\odot}$ take place in the lower part, and those with $M_{\mathrm{A}}>3.4 \mathrm{M}_{\odot}$ appear in the upper part of Figure 3. Near the ZAMS of the primary component, $r_{m}$ is about 0.4 for $M_{\mathrm{A}}<3.4 \mathrm{M}_{\odot}$ and 0.6 for $M_{\mathrm{A}}>3.4 \mathrm{M}_{\odot}$. It increases with time and reaches a value between 1.6 and 2.4. It seems that $r_{m}$ is a better indicator of binary system age than $l_{m}$. For example, the age of a system with $r_{m}=1$ is about half of the TAMS age of the primary component. For more accurate ages, two fits for the two mass ranges are made to the curves in Figure 3.

\subsubsection{DEBs with Relatively Low-mass Primary Component $\left(\mathrm{M}_{A}<3.4 \mathrm{M}_{\odot}\right)$}

In order to increase the precision of Eq. (5), we derive two expressions for low and high values of $r_{m}$.

For the three binary systems with $M_{\mathrm{A}}<3.4 \mathrm{M}_{\odot}$, namely, V618 Per, V1229 Tau (HD23642) and GV Car, three separate fits are made to the curves in Figure 3 by using Eq. (5). Then, the mean values of the parameters ( $a$, $b$ and $n$ ) given in Eq. (5) yield

$$
\frac{t}{t_{\mathrm{MSA}}}=0.941\left(r_{m}-0.39\right)^{0.814} \text { for } r_{m}<1.0
$$

and

$$
\frac{t}{t_{\mathrm{MSA}}}=0.77\left(r_{m}-0.588\right)^{0.431} \text { for } r_{m}>1.0 \text {. }
$$

The ages of V1229 Tau and GV Car computed from these expressions $\left(t_{\mathrm{rmf}}\right)$ are given in the ninth column of 
Table 2. They are in good agreement with the ages found by other methods.

\subsubsection{DEBs with Relatively High-mass Primary Component $\left(\mathrm{M}_{A}>3.4 M_{\odot}\right)$}

Using Eq. (5), two fits are applied to the curves of V615 Per, V497 Cep and V453 Cyg given in Figure 3 according to the value of $r_{m}$. We compute the average values of the parameters $a, b$ and $n$. From these values, the expressions for age as a function of $r_{m}$ are obtained:

$$
\frac{t}{t_{\mathrm{MSA}}}=1.011\left(r_{m}-0.561\right)^{0.995} \text { for } r_{m}<1.0
$$

and

$$
\frac{t}{t_{\mathrm{MSA}}}=0.613\left(r_{m}-0.852\right)^{0.330} \text { for } r_{m}>1.0
$$

For V615 Per, $r_{m}=0.747$ and Eq. (8) gives an age of 27.1 My. These two expressions are used to determine age of the binary systems whose $M_{\mathrm{A}}$ is greater than $3.4 \mathrm{M}_{\odot}$ (see Table 2).

The fitting formula given in Eqs. (6-8) are not valid for V381 Car because of the large mass difference between its components. They are also not applicable to binaries with very similar components. For V392 Car, for example, the mass $(2.47 \%)$ and radius $(1.54 \%)$ differences between its components are so low that the fitting formula gives an age two times greater than the ages found by other methods. The situation for DW Car is better than V392 Car. The mass $(6.26 \%)$ and radius $(5.73 \%)$ differences between the components of DW Car are big enough to predict a reasonable value for age. For V906 Sco, however, there is a big difference between the radii of the components which gives so great a value for $r_{m}$ that we deduce that the age is about $t_{\mathrm{rmf}}=t_{\mathrm{MSA}}=253 \mathrm{My}$.

The $r_{m}$ curve of V1034 Sco $(\times$ in Figure 3$)$, which has the highest primary mass $\left(M_{\mathrm{A}}=16.84 \mathrm{M}_{\odot}\right)$ of the stars studied, is slightly different from the rest. It is in the upper part during the early MS phase as that of the other binaries with $M_{\mathrm{A}}>3.4 \mathrm{M}_{\odot}$, and joins later on the curves for binaries with $M_{\mathrm{A}}<3.4 \mathrm{M}_{\odot}$. Therefore, the expressions given in Eqs. (6-7) shoul be used rather than Eqs. (8-9) for $M_{\mathrm{A}}>15 \mathrm{M}_{\odot}$ when the relative age is greater than 0.5 .

The new expressions proposed above were additionally tested for selected eclipsing binaries. The age of HD 42401 is given by Williams (2009) as $25 \mathrm{My}$ and we find the same age $(25.6 \mathrm{My})$ from the ratio of radii. For the age of V539 Ara Clausen et al. (1996) and Lastennet and Valls-Gabaud (2002) give 45 and $39 \mathrm{My}$, respectively. The ratio of radii gives the age as $40.0 \mathrm{My}$.

\subsection{Age Determination from the Slope of the Mass- Luminosity Relation}

We also derive an expression to determine age from the ratio of luminosities:

$$
t=0.794\left(l_{m}-4.535 M_{\mathrm{A}}^{-0.154}\right) 10 \cdot^{\left(\log M_{\mathrm{A}}-0.9\right)^{2}} t_{\mathrm{MSA}} .
$$

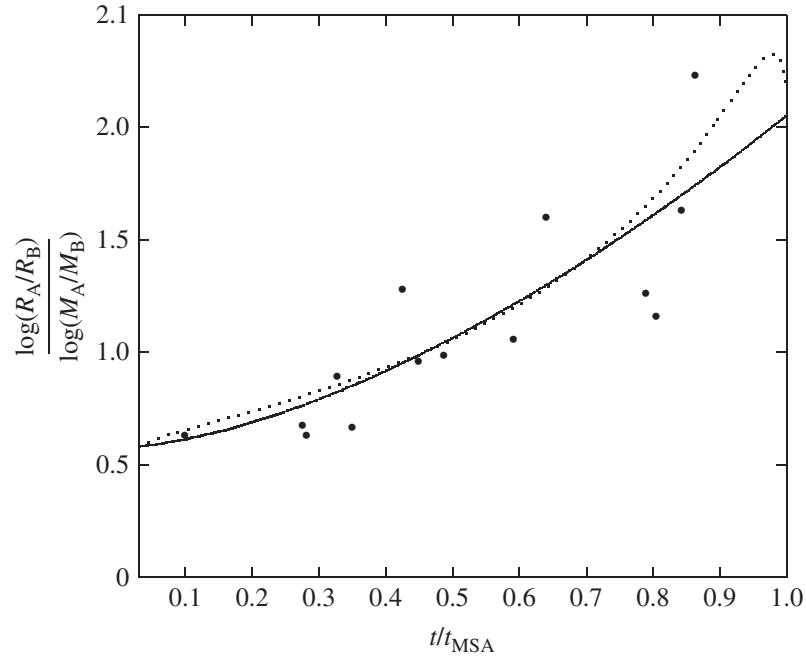

Figure 6 The mass-radius relation for the DEBs given in Andersen (1991) with respect to the relative age of the primary components. The ages are taken from Pols et al. (1997). The solid line is for the curve fitted to this data by using Eq. (5). For comparison, $r_{m}$ of V497 Cep from models (dotted line) is also plotted.

This expression gives acceptable ages for only five of the binary systems (V453 Cyg, V497 Cep, V381 Car, V1034 Sco and GV Car). The reason might be that the ratio of luminosities of component stars derived from the light curve of a binary system may not be as accurate as the ratio of radii. The dependence of $l_{m}$ on $M_{\mathrm{A}}$ is also as important as its time dependence (see Figure 2).

\subsection{Does an $\mathrm{r}_{\mathrm{m}}$-Relative Age Relation Implicitly Exist in the Literature?}

The time dependence of $r_{m}$ is not considered in the previous studies in the literature. However, this dependence also exists in Pols et al. (1997), for example, though the authors are not aware of it. In Figure $6, r_{m}$ is plotted with respect to the ages they find (in units of MS lifetime of the primary components) for the well-known binaries (Andersen 1991). In order to avoid complications, the binaries with $M_{\mathrm{A}}>1.5$ and significantly different components (difference between masses of the components is greater than $10 \%$ ) are considered. The mass range is $1.56-$ $9.245 \mathrm{M}_{\odot}$. The solid line shows the fitted curve for this data using Eq. (5). The dotted line represents the $r_{m}$ of V497 Cep, already plotted in Figure 3. The agreement between the two curves is amazing.

\section{The Uncertainty in the Ages of the Binaries}

The ages of stars that we have found involve many uncertainties, based on the model ingredients (e.g. opacity, nuclear reaction rates, equation of state) and the observational quantities. It is very difficult to compute the uncertainty due to the former and therefore we take care in calculating the uncertainties resulting from the latter in our error analysis. The uncertainty in the age $(\Delta t)$ derived from the luminosities and radii of component stars is the time interval in which the evolutionary tracks of both 
Table 7. The uncertainties in the ages of the binaries derived from the error boxes in the luminosity-radius diagram and the uncertainties in the ratio of the radii of the components

\begin{tabular}{|c|c|c|c|c|c|c|c|}
\hline Star & $t(\mathrm{y})$ & $\Delta t(\mathrm{y})$ & $t(\mathrm{y})$ & $\Delta r_{\mathrm{AB}}$ & $\Delta t_{\mathrm{rel}}$ & $\Delta t_{\mathrm{rm}}(\mathrm{y})$ & Cluster \\
\hline V615 Per & $2 \times 10^{7}$ & $0.61 \times 10^{7}$ & $2.92 \times 10^{7}$ & 0.0836 & 0.712 & $9.96 \times 10^{7}$ & NGC 869 \\
\hline V618 Per & $2 \times 10^{7}$ & $0.61 \times 10^{7}$ & - & - & - & - & $\mathrm{NGC} \times 869$ \\
\hline V453 Cyg & $1.05 \times 10^{7}$ & $0.10 \times 10^{7}$ & $9.76 \times 10^{6}$ & 0.0193 & 0.018 & $0.02 \times 10^{7}$ & NGC 6871 \\
\hline V1229 Tau & $1.93 \times 10^{8}$ & $0.17 \times 10^{8}$ & $1.24 \times 10^{8}$ & 0.0045 & 0.027 & $0.19 \times 10^{8}$ & Pleiades \\
\hline V578 Mon & $3.50 \times 10^{6}$ & $0.14 \times 10^{6}$ & - & - & - & - & NGC 2244 \\
\hline V906 Sco & $2.52 \times 10^{8}$ & $0.21 \times 10^{8}$ & $2.53 \times 10^{8}$ & 0.0241 & 0.069 & $0.17 \times 10^{8}$ & NGC 6475 \\
\hline V497 Cep & $2.30 \times 10^{7}$ & $0.48 \times 10^{7}$ & $2.17 \times 10^{7}$ & 0.0209 & 0.053 & $0.26 \times 10^{7}$ & NGC 7160 \\
\hline V381 Car & $1.05 \times 10^{7}$ & $0.11 \times 10^{7}$ & $1.05 \times 10^{7}$ & 0.0800 & 0.030 & $0.04 \times 10^{7}$ & NGC 3293 \\
\hline V392 Car & $1.23 \times 10^{8}$ & $0.15 \times 10^{8}$ & $1.41 \times 10^{8}$ & 0.0089 & 0.472 & $4.86 \times 10^{8}$ & NGC 2516 \\
\hline V1034 Sco & $5.51 \times 10^{6}$ & $0.42 \times 10^{6}$ & $5.36 \times 10^{6}$ & 0.0080 & 0.016 & $0.16 \times 10^{6}$ & NGC 6231 \\
\hline DW Car & $5.22 \times 10^{6}$ & $0.30 \times 10^{6}$ & $4.88 \times 10^{6}$ & 0.0229 & 0.188 & $2.93 \times 10^{6}$ & $\mathrm{Cr} 228$ \\
\hline GV Car & $3.22 \times 10^{8}$ & $0.61 \times 10^{8}$ & $3.21 \times 10^{8}$ & 0.0406 & 0.064 & $0.27 \times 10^{8}$ & NGC 3532 \\
\hline
\end{tabular}

components stay within the corresponding error boxes in the luminosity-radius ( $\mathrm{L}-\mathrm{R})$ diagram. The values of $\Delta t$ for the binaries are given in the third column of Table 7 . For a binary system whose age is derived from its primary component, the uncertainty is also computed from that star. Within the context of our approach, the uncertainties are reasonably small.

We also compute uncertainties for the ages $\left(\Delta t_{\mathrm{rm}}\right)$ derived from the ratio of the radii $\left(r_{\mathrm{AB}}=R_{\mathrm{A}} / R_{\mathrm{B}}\right)$. The uncertainty in the mass ratio appearing in the denominator of $r_{\mathrm{rm}}$ is usually very small for well-measured binary systems. The values of $\Delta r_{\mathrm{AB}}$ are available in the literature and given in the fifth column of Table 7 . The error in age due to uncertainty in the ratio of the radii is given in the seventh column of Table 7 . The uncertainty in the relative age $\left(\Delta t_{\text {rel }}=\Delta t / t_{\mathrm{MSA}}\right)$ derived from the ratio of radii $\left(r_{\mathrm{AB}}=R_{\mathrm{A}} / R_{\mathrm{B}}\right)$ is computed using

$$
\Delta t_{\mathrm{rel}}=\frac{\Delta r_{\mathrm{AB}}}{\left(\partial r_{\mathrm{AB}} / \partial t_{\mathrm{rel}}\right)} .
$$

The derivative of the ratio of radii with respect to the relative age in Eq. (11) is obtained from the models around the relative age of each binary system. As the masses of the component stars are close to each other, the derivative is negligibly small and therefore the uncertainty in age is very large. This is the case for the binary systems whose components are very similar: V906 Sco, V392 Car and DW Car. Except V615, the rest of the binary systems have very small uncertainties for their ages. For V615 Per, the reason for the large uncertainty is the large value of $\Delta r_{\mathrm{AB}}$.

\section{Conclusions}

Our current understanding of the evolution of our far and near universe depends on accurate age estimates for astrophysical systems. The stars are the main constituents of these systems, whose ages are essentially determined from comparison of their observed and model properties. Rotating (for DW Car A and B) and non-rotating models for the components of the eclipsing binaries, members of open clusters, are constructed to derive the ages and chemical compositions of the binaries and hence the clusters. The largest difference between the ages we found for the eclipsing binaries and the ages given by Kharchenko et al. (2005) for the clusters occurs for the V497 Cep binary system in NGC 7160, where the difference is about $100 \%$. For the remaining systems the difference is less than $40 \%$.

Binary systems such as V906 Sco in NGC6475 and V453 Cyg in NGC 6871 are the most suitable systems for age determination: their primary components are about to complete their MS lifetime and the secondary components are near or not far from the ZAMS. For such systems, despite equivalent solutions with very different chemical compositions, the ages are very close. For the three different solutions with metallicity $0.014-0.017$, the age of V906 Sco is in the range 252-254 My.

The slope of the mass-luminosity relation for a binary system depends on the mass of its primary component $M_{\mathrm{A}}$, if $M_{\mathrm{A}}$ is less than about $10 \mathrm{M}_{\odot}$. The value of $l_{m}=\log \left(L_{\mathrm{A}} / L_{\mathrm{B}}\right) / \log \left(M_{\mathrm{A}} / M_{\mathrm{B}}\right)$ near the ZAMS is 4 for $M_{\mathrm{A}}=2.5 \mathrm{M}_{\odot}, 3.5$ for $M_{\mathrm{A}}=6.89 \mathrm{M}_{\odot}$ and it drops to a minimum value of 3 for $M_{\mathrm{A}}=10 \mathrm{M}_{\odot}$. For binary systems with $M_{\mathrm{A}}$ greater than $10 \mathrm{M}_{\odot}, l_{m}=3$ near the ZAMS and reaches 4.3 near the TAMS age of their primary component.

The slope of the mass-radius relation changes with time more meaningfully than the slope of the massluminosity relation, so is more suitable for estimating the age of the system once it is calibrated. If $r_{m}=1$ for a system, its age is about half the MS lifetime of its primary (massive) component. We have derived simple expressions to estimate the age of a binary system from the slope of the mass-radius and mass-luminosity relations. In particular, the expressions for the slope of the massradius relation give very reasonable results $\left(t_{\mathrm{rm}}\right)$. Eqs. (6-9) can be used to predict the age of any binary system if the radii and masses of its components are known. The new expressions are tested for the eclipsing binaries HD 42401 and V539 Ara and give excellent results for their ages. The advantage of these expressions for the relative age $\left(t / t_{\mathrm{MSA}}\right)$ is that they are independent of chemical composition. 


\section{Acknowledgments}

This work is supported by the Scientific and Technological Research Council of Turkey (TÜBİTAK).

\section{References}

Alencar, S. H. P., Vaz, L. P. R. \& Helt, B. E., 1997, A\&A, 326, 709 Andersen, J., 1991, A\&AR, 3, 91

Asplund, M., Grevesse, N. \& Sauval, A. J., 2005, in ASP Conf. Ser. 336, Cosmic Abundances as Records of Stellar Evolution and Nucleosynthesis, Eds. Bash, F. N. \& Barnes, T. G. (Astron. Soc. Pac.: San Francisco), 25 (AGS2005)

Bouzid, M. Y., Sterken, C. \& Pribulla, T., 2005, A\&A, 437, 769

Clausen, J. V., Garcia, J. M., Gimenez, A., Helt, B. E., Jensen, K. S., Suso, J. \& Vaz, L. P. R., 1996, A\&A, 308, 151

Debernardi, Y. \& North, P., 2001, A\&A, 374, 204

del Peloso, E. F., da Silva, L., Porto de Mello, G. F. \& Arany-Prado, L. I., 2005, A\&A, 440, 1153

Eggenberger, P., Miglio, A., Carrier, F., Fernandes, J. \& Santos, N. C., 2008, A\&A, 482, 631

Freyhammer, L. M., Hensberge, H., Sterken, C., Pavlovski, K., Smette, A. \& Ilijic, S., 2005, A\&A, 429, 631

Freyhammer, L. M., Hensberge, H., Sterken, C., De Cat, P. \& Aerts, C., 2006, Mem. S.A.It., 77, 334

Garcia, E., Stassun, K. G., Hebb, L. \& Heiser, A., 2010, AAS, 21541940

Girardi, L., Bertelli, G., Bressan, A., Chiosi, C., Groenewegen, M. A. T., Marigo, P., Salasnich, B. \& Weiss, A., 2002, A\&A, 391, 195

Groenewegen, M. A. T., Decin, L., Salaris, M. \& De Cat, P., 2007, A\&A, 463, 579
Hensberge, H., Pavlovski, K. \& Verschueren, W., 2000, A\&A, 358, 553

Kharchenko, N. V., Piskunov, A. E., Röser, S., Schilbach, E. \& Scholz, R.-D., 2005, A\&A, 438, 1163

Lastennet, E. \& Valls-Gabaud, D., 2002, A\&A, 396, 551

Mamajek, E. E. \& Hillenbrand, L. A., 2008, ApJ, 687, 1264

Meynet, G. \& Maeder, A., 2000, A\&A, 361, 101

Munari, U., Dallaporta, S., Siviero, A., Soubiran, C., Fiorucci, M. \& Girard, P., 2004, A\&A, 418, 31

Pavlovski, K. \& Hensberge, H., 2005, A\&A, 439, 309

Pavlovski, K. \& Southworth, J., 2009, MNRAS, 394, 1519

Pols, O. R., Tout, C. A., Schroder, K.-P., Eggleton, P. P. \& Manners, J., 1997, MNRAS, 289, 869

Schaller, G., Schaerer, D., Meynet, G. \& Maeder, A., 1992, A\&AS, 96, 269

Southworth, J., Maxted, P. F. L. \& Smalley, B., 2004a, MNRAS, 349,547

Southworth, J., Maxted, P. F. L. \& Smalley, B., 2004b, MNRAS, 351,1277

Southworth, J., Maxted, P. F. L. \& Smalley, B., 2005, A\&A, 429, 645

Southworth, J. \& Clausen, J. V., 2006, Ap\&SS, 304, 199

Southworth, J. \& Clausen, J. V., 2007, A\&A, 461, 1077

Torres, G., Andersen, J. \& Gimenez, A., 2010, A\&ARv, 18, 67

Williams, S. J., 2009, AJ, 137, 3222

Yakut, K., Tarasov, A. E., İbanoğlu, C., Harmanec, P., Kalomeni, B., Holmgren, D. E., Bozic, H. \& Eenens, P., 2003, A\&A, 405, 1087 Yıldız, M. \& Kızıloğlu, N., 1997, A\&A, 362, 187

Yildiz, M., 2003, A\&A, 409, 689

Yildı, M., 2005, MNRAS, 363, 967

Yıldı,, M., Yakut, K., Bakıș, H. \& Noels, A., 2006, MNRAS, 368, 1941

Y1ldiz, M., 2007, MNRAS, 374, 1264

Y1ldı, M., 2008, MNRAS, 388, 1143 